\title{
The Protective Effect of Matricaria chamomilla Extract on Histological Damage and Oxidative Stress Induced by Torsion/Detorsion in Adult Rat Ovary
}

\author{
Malihe Soltani ${ }^{1}$, Maryam Moghimian $^{1 *}$, Hosein Abtahi ${ }^{1}$, Majid Shokoohi ${ }^{2,3}$
}

\begin{abstract}
Objectives: This experimental study used a rat model to investigate the effect of Matricaria chamomilla (MC) extract on histological damage and oxidative stress induced by torsion/detorsion in adult rat ovary.

Materials and Methods: In our experimental study, 28 female Wistar rats were used. They were randomly divided into 4 groups; G1, Sham group; G2, Ovarian torsion for 3 hours followed by detorsion 10 days (TDO); G3, Ovarian torsion for 3 hours and then received $200 \mathrm{mg} / \mathrm{kg}$ hydroalcoholic extracts of MC, 30 minutes before detorsion, followed by detorsion (10 days) (TDOMC); G4, received $200 \mathrm{mg} / \mathrm{kg}$ of hydroalcoholic extracts of MC. After the 10-day reperfusion period, blood sampling was done and blood levels of estrogen, testosterone, some oxidative stress markers and anti-oxidant enzymes were assayed. Further assessment was carried out by histomorphometry on 10-day postprocedure.

Results: Comparison of the histological parameters showed a significant change in the G2 group as compared with other groups. The levels of estrogen, glutathione peroxidase (GPx), and superoxide dismutase (SOD) significantly decreased in G2 group, and increased in G3 and G4 groups, and the malondialdehyde level increased in the duration of ischemia. Treatment by MC decreased the malondialdehyde level in G3 and G4 groups.

Conclusion: The results of this study showed that MC could reduce oxidative stress and tissue damages following ovarian torsion/ detorsion.

Keywords: Matricaria chamomilla, Ovary, Oxidative stress, Tissues, Torsion
\end{abstract}

\section{Introduction}

Ovarian torsion is one of the prevalent gynecologic emergencies, in which the adnexal components swing around their axis that leads to obstruction of arterial, venous, and/or lymphatic vessels (1). In this situation, gangrene and necrosis with the expansion of arterial insufficiency is followed by primary congestion and hemorrhagic infarction owing to venous and lymphatic obstruction. However, there is no data about the least number of turns to form the torsion and also there is no data for the duration of torsion that is necessary for the setting in of necrosis (2). Diagnosis of torsion is based on clinical indication and found by sonographic findings. Meanwhile a definite diagnosis is made only during surgery (1) Oophorectomy of the necrotic and/ or doubtful ovaries after torsion or incarceration is the preferred surgical treatment in contemporaneous practice. However, before maturity, the importance of losing the gonads is higher than its loss in later periods. Also, nonsynchronous contralateral torsion has an occurrence ranging between $2 \%-5 \%$, therefore, is considered as a clinical catastrophe. Twenty-five percent of children with torsion have abnormal ovaries. Moreover, protection of the gonads is the main attention in children. Therefore, a more conservative approach has been recommended in the last research (2).

In the past, remedial action to overcome ovarian torsion was surgical removal of the ovaries. Nowadays, due to the importance of the ovaries in fertility and secretion of sexual hormones, ovaries are retained (3). Ovarian torsion/detorsion or ischemia/reperfusion of ovary is a pathophysiologic event in which histological damage is associated with perfusion reduction followed by lack of oxygen (ischemia) in ovarian tissue. The inflammatory response induced by torsion/detorsion (reperfusion) can lead to vascular endothelial cells damage and microcirculation disorders that are largely responsible for the injury to the ovarian tissue. The damage caused by ischemia/reperfusion produces excessive reactive oxygen species (ROS) such as hydroxyl free radical species, hydrogen peroxide $\left(\mathrm{H}_{2} \mathrm{O}_{2}\right)$, and superoxide radical (46). Therefore, oxidation has wrecking effects on ovarian tissue, thus, antioxidant substances not only have great roles in the recovery process but also reverse the adverse effects. Natural products such as plant extracts are a good alternative for drugs used in oxidative damage (7).

Received 12 December 2016, Accepted 8 June 2017, Available online 1 July 2017

${ }^{1}$ Department of Basic Sciences, Faculty of Medicine, Gonabad University of Medical Sciences, Gonabad, Iran. ${ }^{2}$ Student Research Committee, Gonabad University of Medical Sciences, Gonabad, Iran. ${ }^{3}$ Department of Anatomical Sciences, Faculty of Medicine, Tabriz University of Medical Sciences, Tabriz, Iran.

*Corresponding Author: Maryam Moghimian, Tel: +985157225813, Email: moghimian.m@gmu.ac.ir 
Chamomile is a perennial plant from Asteraceae family, growing in West Europe and North Africa. In traditional medicine chamomile tea is used for treating stomach disturbances with antispasmodic and anti-inflammatory effects. In women, the antispasmodic effects of chamomile can lessen the menstrual cramps and detract the possibility of preterm delivery. Also it stimulates the menstruation (8).

Several studies on chamomile extract showed that both the lipophilic and hydrophilic forms, are effective in therapeutic activities. The most constituents of chamomile are unstable oils, sesquiterpene lactones, and phenolic compounds including flavonoids. The substantial compounds of chamomile flowers contain different phenolic compounds, primarily the flavonoids, apigenin, quercetin, patulin, luteolin, and glycosides (8). Flavonoids are chemical phenyl benzopyrones which are usually observed in all vascular plants. The benzopyran on ring system is a molecular scaffold which can be seen in flavonoid as inherent products and also acclaimed it has weak aromatase inhibitory activity (8). The experimental and clinical studies performed on chamomile concluded that its main pharmacological functions are related to its antioxidant activity. It is mostly due to its capability to control the free radicals and/or inhibit lipid peroxidation $(9,10)$. However, according to anti-inflammatory and antioxidant effects of this plant, we decided to use chamomile to reduce tissue damage and oxidative stress caused by torsion/detorsion in the adult rat ovaries.

\section{Materials and Methods}

For the present study, 28 female Wistar rats with average weight of 200-250 g were used. Animals were purchased from the Mashhad Razi Institute and kept in the same place at standard conditions (temperature $25^{\circ} \mathrm{C}$, and 12 $\mathrm{h} / 12 \mathrm{~h}$ light/dark cycle). During the experimental period, rats had free access to food and water. All experimental steps of the present study were conducted in accordance with Gonabad University of Medical Science Guidelines for the Care and Use of Laboratory Animals.

\section{Animal}

Rats were randomly divided into 4 groups. G1, Sham group, a longitudinal cutting $(2.5 \mathrm{~cm})$ was made in the midline region of the lower abdomen, then the incision was closed with $5 / 0$ nylon sutures $(n=7)$.

G2, ovarian torsion for 3 hours. Also, they received normal saline by oral gavage 30 minutes before detorsion. Detorsion duration was 10 days and rats received normal saline by oral gavage (OT) $(n=7)$.

G3, ovarian torsion for 3 hours and rats received 200 $\mathrm{mg} / \mathrm{kg}$ hydroalcoholic extracts of Matricaria chamomilla (MC) by oral gavage 30 minutes before detorsion. Detorsion duration was 10 days and rats received MC extract by oral gavage (OTMC) $(n=7)$.

G4 or MC group in which no operation was done. But, all the rats $(\mathrm{n}=7)$ received $200 \mathrm{mg} / \mathrm{kg}$ hydroalcoholic extract of MC by oral gavage for 10 days (daily). After 10 days, the left ovary was removed for histological studies. The purpose of this group study was an investigation of side effects of MC extract $(200 \mathrm{mg} / \mathrm{kg})$ on histological parameters of the ovaries and biochemical factors of blood $(n=7)$.

\section{Surgical Procedure and Sampling}

After the acclimatization period, animals were anesthetized by ketamine $(50 \mathrm{mg} / \mathrm{kg})$ and xylazine $(10 \mathrm{mg} / \mathrm{kg})$. Then, a longitudinal cutting $(2.5 \mathrm{~cm})$ was made in the central region of the lower abdomen. A small peritoneal cutting was performed, and the left uterine horns and adnexa were revealed. Afterward, the left ovary was rotated 720 degrees in a clockwise direction around its axis and then to avoid detorsion of it, fixed to the abdominal wall with 0.6 nylon. Then, the incision was closed with 5/0 nylon sutures that kept the torsion for 3 hours. Thirty minutes before the opening of ovarian torsion, the chamomile extract was administered orally. After the end of the ischemic period ( 3 hours), the twisting of the ovary opened, then 10 days were allowed to ovaries for reperfusion. At the end of reperfusion, rats in all groups were anesthetized with the ketamine xylazine, then the blood samples were taken from the heart to evaluate metabolic changes. Also, ovarian tissue was removed to evaluate the histological changes $(8,11)$. In order to fixate the ovaries, samples were placed in $10 \%$ formalin for 72 hours, then dehydrated and embedded in paraffin and preprepared the histological slides with $5 \mu \mathrm{m}$ cutting by microtome. Finally, all species were stained by hematoxylin-eosin ( $\mathrm{H} \& \mathrm{E})$. The blood samples were centrifuged for 5 minutes at $4000 \mathrm{rpm}$, then the serum was separated and each of serum samples poured in 3 microtubes $(500 \mu \mathrm{L})$ and transferred to the freezer $-70^{\circ} \mathrm{C}$ until the time of experiment.

\section{Histological Study}

To perform histological and histometrical studies, we examined tissue sections of each ovary from the cortex to the medulla in a spirally and clockwise direction. In each slide, the number of primordial, primary, antral, and graafian follicles as well as atretic and yellow bodies were counted. Also, they were compared between different groups.

Evaluation of Biochemical Parameters

Malondialdehyde (MDA) levels was measured by placing $0.20 \mathrm{~cm}^{3}$ of plasma into a test tube containing $3.0 \mathrm{~cm}^{3}$ of glacial acetic acid, to which $3.0 \mathrm{~cm}^{3}$ of $1 \%$ TBA in $2 \%$ $\mathrm{NaOH}$ was added. The test tube containing the mixture was placed in boiling water for 15 minutes. The absorbance of the pink-colored product was read at $532 \mathrm{~nm}$ after cooling. The calibration curve was constructed using malondialdehyde tetrabutyl ammonium salt obtained from Sigma (USA).

Measurement of the Activity of Superoxide Dismutase and Glutathione Peroxidase

Superoxide dismutase (SOD) and glutathione peroxidase 
(GPx) activity in serum were assayed in accordance with the protocols of the kits used (Randox, UK).

Measurement of Testosterone and Estrogen Level The serum hormone levels were determined using a testosterone and estrogen enzyme-linked immunosorbent assay (ELISA) kit (Demeditec Diagnostics, Germany). Absorbance was measured at $405 \mathrm{~nm}$ for testosterone and estrogen.

\section{Statistical Analysis}

Statistical analysis was done in SPSS 20 (IBM, USA). The Kolmogorov-Smirnov test was used to determine whether or not the data were normally distributed. All data were presented as mean \pm standard error (SE). One-way analysis of variance (ANOVA) followed by Tukey post hoc test was performed to compare the histopathological parameters and oxidative stress values. Statistical significance levels were determined at $P<0.05$.

\section{Results}

Histological Parameters of Ovarian Tissue

The Number of Follicles

Comparison of the number of follicles showed a significant decrease in the OT group rather than the sham group $(P<0.05)$. Also, a significant difference between OT and OTMC groups and between OT and MC groups was observed $(P<0.05)$. The number of primary follicles was significantly decreased in the OT groups as compared with the sham group $(P<0.05)$. On the other hand, a significant difference between OT group and other experimental groups $(P<0.05)$ were observed. The number of antral follicles was significantly decreased in the OT groups as compared with the sham group $(P<0.05)$. Also, in OTMC and MC groups, the number of antral follicles was significantly increased as compared with OT group $(P<0.05)$. The number of graafian follicles was significantly reduced in the OT groups as compared with the sham group $(P<0.05)$. On the other hand, the number of graafian follicles was increased in the OTMC and $\mathrm{MC}$ groups as compared with the OT group $(P<0.05)$. Comparison between the number of atretic bodies in different groups showed that the atretic bodies were significantly increased in the OT group as compared with the sham group $(P<0.05)$. Also, in OTMC and MC groups, the number of atretic bodies was significantly decreased as compared with OT group $(P<0.05)$. The number of yellow bodies was significantly decreased in OT group as compared with sham group $(P<0.05)$. But, in the OTMC and MC groups, the number of yellow bodies was significantly increased as compared with OT group $(P<0.05)$ (Table 1; Figure 1).

The Serum Level of Estrogen

The serum level of estrogen was significantly decreased in OT group as compared with the sham group $(\mathrm{p}<0.05)$. Also, the estrogen level in OTMC and MC groups was significantly increased when compared with the OT group $(P<0.05)$ (Figure 2A).

The Serum Level of Testosterone

The serum level of testosterone was significantly increased in the OT group as compared with the sham group $(P<0.05)$. On the other hand, a significant difference between OT group with OTMC and MC groups was observed $(P<0.05)$ (Figure 2B).

The Serum Level of Superoxide Dismutase

The serum level of SOD was significantly decreased in OT the group compared with the sham group $(P<0.05)$. Also, in OTMC and MC groups, the serum SOD level was significantly increased as compared with the OT group
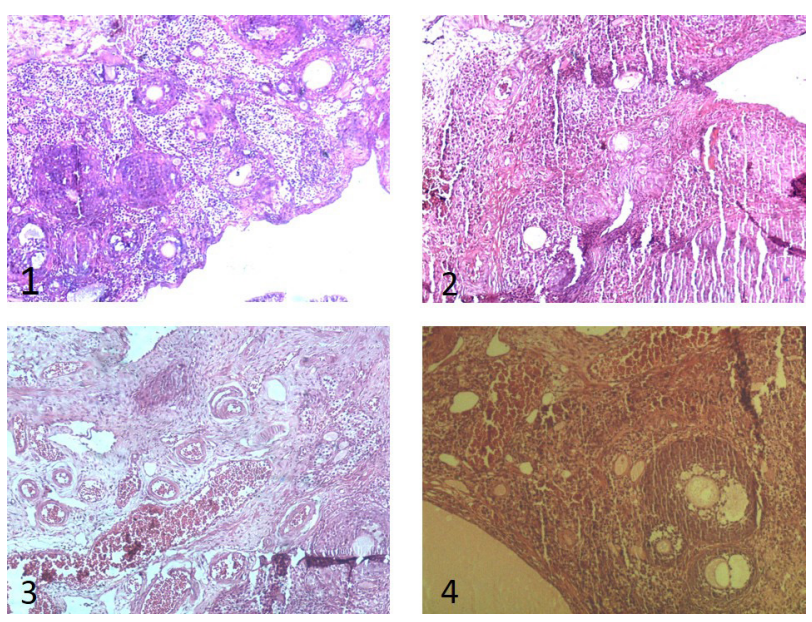

Figure 1. Histological Findings in Sham, OT, OTMC and MC Group 24 hours After Surgery

Table 1. The Mean Number of Primordial, Primary, Antral, and Graafian Follicles and Atretic and Yellow Bodies in the Ovaries of Rats in the Experimental and Control Groups

\begin{tabular}{lcccccc}
\hline Group & Primordial Follicles & Primary Follicles & Antral Follicles & Graafian Follicles & Yellow Bodies & Atretic Bodies \\
\hline Sham & $8.2 \pm 3.72$ & $5.92 \pm 1.21$ & $6.7 \pm .42$ & $5.2 \pm 0.61$ & $5.3 \pm 0.27$ & $2.3 \pm 0.11$ \\
OT & $1.5 \pm 1.05^{*}$ & $1.07 \pm 0.702^{*}$ & $2.23 \pm .72^{*}$ & $1.2 \pm 0.21^{*}$ & $3.01 \pm .41^{*}$ & $6.2 \pm 0.51^{*}$ \\
OTMC & $5.4 \pm 2.1 \dagger^{*}$ & $4.3 \pm 1.30 \dagger$ & $5.03 \pm 1.2 \dagger$ & $4.07 \pm 0.74 \dagger$ & $4.7 \pm 0.34 \dagger$ & $3.07 \pm 034 \dagger$ \\
MC & $8.5 \pm 2.3 \dagger$ & $6.01 \pm 2.70 \dagger$ & $6.9 \pm 1.7 \dagger$ & $5.62 \pm 0.62 \dagger$ & $4.9 \pm 0.82 \dagger$ & $2.62 \pm 0.82 \dagger$ \\
\hline
\end{tabular}

Note: The asterisk sign $(*)$ shows significant difference with the sham group and the symbol of + means the significant difference with the OT group $(P<0.05)$. 
A

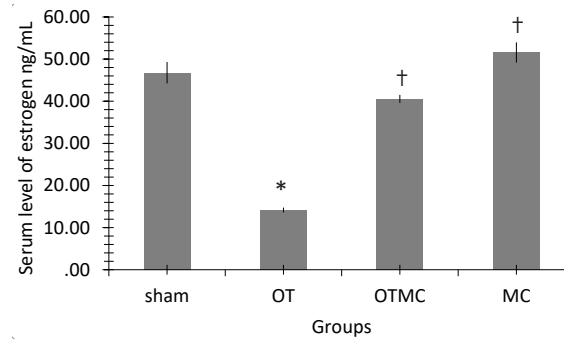

C

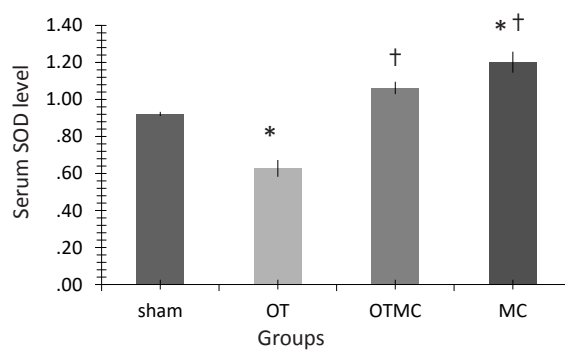

E

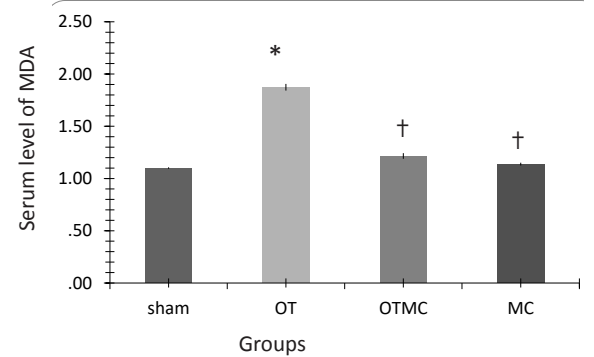

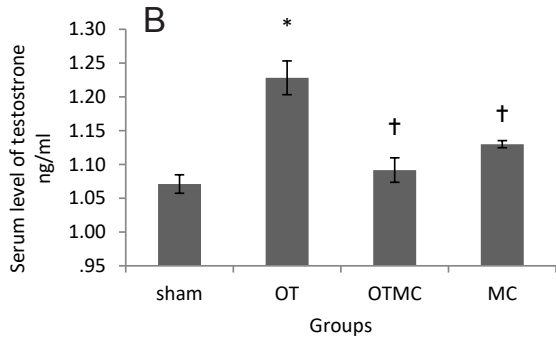

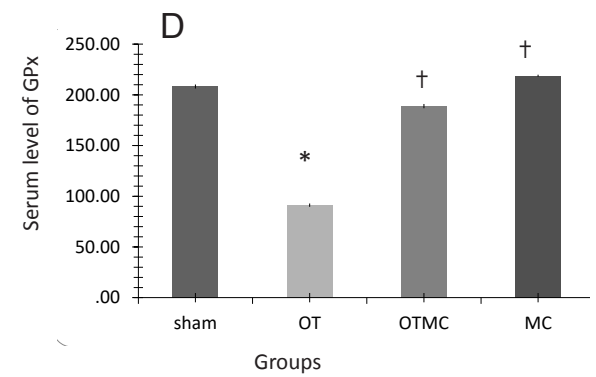

Figure 2. Comparison of the serum level of (A) estrogen, (B) testosterone, (C) SOD, (D) GPx, and (E) MDA in different groups.

$(P<0.05)$ (Figure 2C).

The Serum Level of Glutathione Peroxidase

The serum level of GPx was significantly decreased in OT group compared with the sham group $(P<0.05)$. On the other hand, a significant difference between OT group to OTMC and MC groups was observed $(P<0.05)$ (Figure 2D).

The Serum Level of Malondialdehyde

The serum level of MDA was significantly increased in OT group compared with the sham group $(P<0.05)$. On the other hand, in OTMC and MC groups, the serum MDA level was significantly decreased as compared with the OT group $(P<0.05)$ (Figure $2 \mathrm{E})$.

\section{Discussion}

In the normal human, ROS and antioxidants stay in equilibrium. The exorbitant productions of ROS lead to the oxidative stress (OS). The complete reproductive lifespan of a woman or even afterward can be affected by OS. OS results in instability between ROS production and capability of the body's defense (antioxidants). ROS have a function similar to a double-edged sword; they act as key signal molecules in many physiological processes of the body but also have an important function in pathological injury such as the female reproductive system.

ROS are effective in several physiological processes from oocyte maturation to fertilization, development of embryo, and pregnancy $(12,13)$. It has been acclaimed that OS has a function in age-related fertility power reduction. It has important functions during gestation, usual childbirth and in initiation of preterm delivery $(12,13)$. Oxidative stress is the base of damage to DNA of the ovarian epithelium and ovulation; it can be hindered by antioxidant compounds. Several studies have shown that OS plays an important role in the pathophysiology of infertility $(12,13)$. The evidence shows that OS has a role in endometriosis, tubal and peritoneal infertility factors $(12,13)$. Antioxidant compounds can be effective in preventing the overproduction of ROS and also can be used to overcome infertility disorders related to the OS $(12,13)$. Many scientific studies showed that ovarian torsion/detorsion or ischemia-reperfusion could be the result of overproduction of ROS followed by the damages caused by OS $(4,6,11)$. Production of OS by ovarian torsion/ detorsion may lead to damage to tissues and hormones such as decreasing the level of estrogen and the serum level of SOD and GPx, reducing the number of follicles, and also increasing the serumlevel of MDA and testosterone $(12,13)$. In the present study, ovarian torsion leads to oxidative damage including tissue and biochemical damages. Decreasing the number of follicles (primordial, primary, antral, and graafian follicles) and yellow bodies, and also increasing the number of atretic bodies, can be considered as a tissue injury. In agreement with our results, Agarwal et al highlighted that ROS leads to decrease in the numbers of primordial, primary, antral, and graafian follicles 
(13). The result of the present study showed that ovarian torsion/detorsion causes biochemical damages such as decreasing the level of estrogen and increasing the level of testosterone. Agarwal et al reported that OS can lead to reduce the level of estrogen (13). Also, Túnez et al indicated that OS can increase the level of testosterone in female rats (14). Our results in this study show that OS induced by ovarian torsion/detorsion can reduce the level of SOD and GPx, while the level of MDA is increased. These results show a decline in antioxidant defense systems. In agreement with our results, Agarwal et al emphasized that ROS leads to decrease in the level of SOD and GPx, also increasing the level of MDA (13). Also, Celik et al showed that ovarian torsion for 3 hours followed by detorsion can decrease the level of GPx and increase the level of MDA and XO (15). On the other hand, Halici et al showed that ovarian torsion for 3 hours and detorsion can reduce the level of SOD (4).

MC extract has several chemical compositions. The most important active components of it include flavonoids, apigenin and alpha-bisabolol (16). According to research results, the compounds of chamomile extract have antiinflammatory, antibacterial and antioxidant activity (17). MC due to its flavonoid and bisabolol compounds is a powerful antioxidant for neutralizing radical oxygen and prevents the formation of free radicals and lipid peroxidation $(18,19)$.

According to this content, we decided to use chamomile extract to reduce the damage of OS caused by ovarian torsion/detorsion. Our results in the present study show that the MC extract by preventing ROS formation and OS can increase the number of follicles and yellow bodies, while the number of atretic bodies is decreased. In agreement with our results, Farideh et al showed that treatment with $\mathrm{MC}$ extract for 10 days leads to increase in the numbers of follicles (20). Our results in the present study show that the MC extract can increase the level of estrogen in rats whose ovaries have undergone torsion, and also by preventing OS, the serum level of testosterone was reduced. This may be due to the presence of apigenin compounds in MC extract. Gradolatto et al (21) showed that the apigenin compounds can increase the level of estrogen, which is in agreement with our results. Results of present research show that MC extract can increase the level of SOD, GPx and reduce the level of MDA and prevent lipid peroxidation, which is related to the presence of the antioxidant compounds in MC extract. In agreement with our results, Chandrashekhar et al showed MC extract can increase the serum level of SOD, GSH and decrease the lipid peroxidation (22). Also, in a separate study, Cemek and colleagues revealed that MC extract can decrease the level of MDA and increase the plasma level of SOD, catalase, and GPX (23).

\section{Conclusion}

According to the results of present study, the MC extract can increase the level of estrogen and protect the tissue damage and OS induced by ovarian torsion/detorsion.

\section{Ethical Issues}

All procedures were conducted in accordance with the ethical committee of Tabriz University of Medical Sciences.

\section{Conflict of Interests}

The authors declare no conflict of interest in this study.

\section{Financial Support}

Student Research Committee of Gonabad University of Medical Sciences supported the study.

\section{Reference}

1. Navve D, Hershkovitz R, Zetounie E, Klein Z, Tepper R. Medial or lateral location of the whirlpool sign in adnexal torsion: clinical importance. J Ultrasound Med. 2013;32(9):1631-1634. doi: 10.7863/ultra.32.9.1631.

2. Kazez A, Ozel SK, Akpolat N, Goksu M. The efficacy of conservative treatment for late term ovarian torsion. Eur J Pediatr Surg. 2007;17(2):110-114.

3. Oelsner G, Shashar D. Adnexal torsion. J Clin Obstet Gynecol. 2006;49(3):459-463.

4. Halici Z, Karaca M, Keles ON, et al. Protective effects of amlodipine on ischemia-reperfusion injury of rat ovary: biochemical and histopathologic evaluation. Fertil Steril. 2008;90(6):2408-2415. doi: 10.1016/j.fertnstert.2007.10.007.

5. Zimmerman BJ, Granger DN. Mechanisms of reperfusion injury. Am J Med Sci. 1994;307(4):284-292.

6. Yuk JS, Kim LY, Shin JY, Choi DY, Kim TY, Lee JH. A national population-based study of the incidence of adnexal torsion in the Republic of Korea. Int J Gynaecol Obstet. 2015;129(2):169-170. doi: 10.1016/j.ijgo.2014.11.027.

7. Komaki E, Yamaguchi S, Kinoshita M, Kakehi K, Ohta Y, Tsukada Y. Identification of anti-alpha-amylase components from olive leaf extracts. Food Sci Technol Res. 2003;9(1):35-39.

8. Farideh ZZ, Bagher M, Ashraf A, Akram A, Kazem M. Effects of chamomile extract on biochemical and clinical parameters in a rat model of polycystic ovary syndrome. J Reprod Infertil. 2010;11(3):169-174.

9. Sebai H, Jabri MA, Souli A, et al. Antidiarrheal and antioxidant activities of chamomile (Matricaria recutita L.) decoction extract in rats. J Ethnopharmacol. 2014;152(2):327-332. doi: 10.1016/j.jep.2014.01.015.

10. Hernández-Ceruelos A, Madrigal-Santillán E, MoralesGonzález JA, Chamorro-Cevallos G, Cassani-Galindo M, Madrigal-Bujaidar E. Antigenotoxic effect of Chamomilla recutita (L.) Rauschert essential oil in mouse spermatogonial cells, and determination of its antioxidant capacity in vitro. Int J Mol Sci. 2010;11(10):3793-3802. doi: 10.3390/ijms11103793.

11. Mostajeran F, Naderi M, Adibi S. Effects of melatonin on histopathological changes after experimental ovarian torsion-detorsion in cat. Int J Reprod Biomed. 2007;5(4):177-182.

12. Agarwal A, Gupta S, Sharma RK. Role of oxidative stress in female reproduction. Reprod Biol Endocrinol. 2005;3:28.

13. Agarwal A, Aponte-Mellado A, Premkumar BJ, Shaman A, Gupta S. The effects of oxidative stress on female reproduction: a review. Reprod Biol Endocrinol. 2012;10:49. doi: 10.1186/1477-7827-10-49.

14. Túnez I, Feijóo M, Collado JA, et al. Effect of testosterone 
on oxidative stress and cell damage induced by 3-nitropropionic acid in striatum of ovariectomized rats. Life Sci. 2007;80(13):1221-1227.

15. Celik O, Turkoz Y, Hascalik S. The protective effect of caffeic acid phenethyl ester on ischemia-reperfusion injury in rat ovary. Eur J Obstet Gynecol Reprod Biol. 2004;117(2):183188.

16. Gardiner P. Complementary, holistic, and integrative medicine: chamomile. Pediatr Rev. 2007;28(4):e16-e18

17. Agatonovic-Kustrin S, Babazadeh Ortakand D, Morton DW, Yusof AP. Rapid evaluation and comparison of natural products and antioxidant activity in calendula, feverfew, and German chamomile extracts. J Chromatogr A. 2015;1385:103-110. doi: 10.1016/j.chroma.2015.01.067.

18. Braga PC, Dal Sasso M, Fonti E, Culici M. Antioxidant activity of bisabolol: inhibitory effects on chemiluminescence of human neutrophil bursts and cellfree systems. Pharmacology. 2009;83(2):110-115. doi: $10.1159 / 000186049$.

19. Sikka SC. Oxidative stress and role of antioxidants in normal and abnormal sperm function. Front Biosci. 1996;1:e78-e86.

20. Farideh ZZ, Bagher M, Ashraf A, Akram A, Kazem M. Effects of chamomile extract on biochemical and clinical parameters in a rat model of polycystic ovary syndrome. J Reprod Infertil. 2010;11(3):169-174.

21. Gradolatto A, Basly JP, Berges R, et al. Pharmacokinetics and metabolism of apigenin in female and male rats after a single oral administration. Drug Metab Dispos. 2005;33(1):49-54.

22. Chandrashekhar VM, Ranpariya VL, Ganapaty S, Parashar A, Muchandi AA. Neuroprotective activity of Matricaria recutita Linn against global model of ischemia in rats. J Ethnopharmacol. 2010;127(3):645-651. doi: 10.1016/j. jep.2009.12.009.

23. Cemek M, Kağa S, Simşek N, Büyükokuroğlu ME, Konuk M. Antihyperglycemic and antioxidative potential of Matricaria chamomilla L. in streptozotocin-induced diabetic rats. J Nat Med 2008;62(3):284-293. doi: 10.1007/ s11418-008-0228-1.

Copyright ( 2017 The Author (s); This is an open-access article distributed under the terms of the Creative Commons Attribution License (http://creativecommons.org/licenses/by/4.0), which permits unrestricted use, distribution, and reproduction in any medium, provided the original work is properly cited. 